\title{
Tracy Shildrick, Robert MacDonald, Colin Webster et Kayleigh Garthwaite, Poverty and insecurity. Life in low-pay, no-pay Britain
}

Bristol, Policy Press, 2013

Philippe Méhaut

\section{OpenEdition \\ Journals}

Édition électronique

URL : http://journals.openedition.org/travailemploi/6670

DOI : 10.4000/travailemploi.6670

ISSN : $1775-416 \mathrm{X}$

Éditeur

DARES - Ministère du Travail

Édition imprimée

Date de publication : 1 avril 2015

Pagination : 104-106

ISSN : 0224-4365

Référence électronique

Philippe Méhaut, «Tracy Shildrick, Robert MacDonald, Colin Webster et Kayleigh Garthwaite, Poverty and insecurity. Life in low-pay, no-pay Britain », Travail et Emploi [En ligne], 142 I avril-juin 2015, mis en ligne le 01 avril 2015, consulté le 23 septembre 2020. URL : http://journals.openedition.org/ travailemploi/6670; DOI : https://doi.org/10.4000/travailemploi.6670

Ce document a été généré automatiquement le 23 septembre 2020.

(c) Direction de l'animation de la recherche, des études et des statistiques (Dares) 


\section{Tracy Shildrick, Robert MacDonald, Colin Webster et Kayleigh Garthwaite, Poverty and insecurity. Life in low-pay, no-pay Britain}

Bristol, Policy Press, 2013

Philippe Méhaut

\section{RÉFÉRENCE}

Tracy Shildrick, Robert MacDonald, Colin Webster et Kayleigh Garthwaite, Poverty and insecurity. Life in low-pay, no-pay Britain, Bristol, Policy Press, 2013, 264 p.

1 En 250 pages, l'ouvrage nous fait plonger au cœur du segment du marché du travail le plus problématique du Royaume-Uni: celui de ces travailleurs «invisibles» qui alternent petits boulots et passages par l'aide sociale, sans vraiment jamais sortir de la pauvreté.

2 Sa force est de s'appuyer sur des entretiens biographiques au long court. Les auteurs, sociologues, juristes, géographes ont enchaîné plusieurs recherches sur le même terrain, retrouvant par exemple douze ans après des jeunes qu'ils avaient enquêtés lors de leurs premiers pas sur le marché du travail. Les matériaux recueillis sont ainsi essentiellement longitudinaux, qualitatifs et biographiques. Les enquêtes ont été menées dans une région (Teesside, dans le Nord-Est de l'Angleterre) de tradition industrielle, marquée par la crise du fordisme et de la grande industrie, au tissu économique profondément déstructuré. Le chômage y est endémique, malgré quelques signes de recomposition. Nul doute que nous pourrions trouver en France des territoires aux caractéristiques très voisines.

3 Cette monographie sur un territoire propose un "carottage» en profondeur, sans prétention a priori à la généralisation mais qui confronte en permanence ses résultats à 
ce que l'on sait dans le monde anglo-saxon, notamment aux États-Unis sur les working poor (travailleurs pauvres). Elle fait aussi écho aux constats de Hugh Frazer et Éric Marlier ${ }^{1}$ sur la segmentation croissante des marchés du travail et l'augmentation d'emplois de mauvaise qualité dans plusieurs pays européens.

En dix chapitres (dont une introduction et une conclusion substantielles), l'ouvrage explore successivement les différentes facettes du cycle bas salaire / absence de salaire - pauvreté récurrente.

5 Il s'ouvre sur un chapitre de discussion de la littérature et des concepts. C'est à l'intersection de quatre cercles que les auteurs se situent. Le premier est celui de l'emploi précaire. Viennent ensuite celui du travail à bas niveau de salaire, celui du cycle bas salaire/absence de salaire que permet d'appréhender la perspective longitudinale, avec le passage par des périodes de pauvreté, et enfin, celui du travail de faible qualité. En la matière, l'étude ne s'intéresse pas simplement au statut d'emploi, mais à l'ensemble des conditions de travail, articulées aux conditions de vie et à la protection sociale.

6 Le chapitre suivant expose la méthodologie. Il présente d'abord les caractéristiques de la région sous revue, son processus de désindustrialisation, la difficulté à voir des signes tangibles de renouveau économique. Il détaille l'articulation des quatre enquêtes qui s'y sont succédé. Les deux premières, réalisées en 1998-1999 et 1999-2001, ciblaient plus particulièrement des jeunes de 15 à 25 ans. La troisième (2003) suivait une partie de ces cohortes, alors âgées de 23 à 29 ans. Une moitié de la cohorte ( 60 personnes) de la dernière vague correspond à des personnes précédemment enquêtées, l'autre moitié incluant des salariés plus âgés. L'une des thèses des auteurs est en effet que le segment des mauvais emplois, en expansion, n'est pas (ou plus) limité à la frange des nouveaux entrants, qu'ils soient jeunes ou migrants. De fait, la pauvreté est partout: trois cinquièmes des interviewés ont connu des épisodes récurrents (au moins deux) de pauvreté alors qu'un cinquième n'est jamais passé au-dessus du seuil de pauvreté ; seul le dernier cinquième comprend des individus n'ayant connu qu'un seul ou aucun épisode de pauvreté.

7 Les auteurs examinent ensuite les pratiques des employeurs et celles des multiples agences, souvent privées, chargées du welfare to work à l'anglaise ${ }^{2}$. Même si les politiques ont varié sur la période étudiée, l'octroi de la plupart des aides s'accompagne d'incitations fortes à se remettre au travail. Dans le même temps, ces dispositifs sont ciblés sur les chômeurs de longue durée, alors que la population étudiée se caractérise plutôt par la récurrence d'épisodes d'emploi (souvent courts) et de chômage. On trouve dans ce chapitre des notations classiques sur les barrières à l'embauche, discriminatoires ou non. Plus étonnant pour le lecteur français (familier du guichet unique - Pôle emploi ou mission locale) est le récit détaillé du passage des enquêtés par une succession d'agences privées (commerciales ou associatives), fonctionnant sur fonds publics alloués sur critères de résultats (principalement le taux de placement). Les auteurs en comptent une douzaine dans un même quartier, avec parfois le même nom. Dans ce maquis, il est difficile de se repérer et de saisir les complémentarités ou les redondances de leur activité.

Une fois le paysage posé, les quatre chapitres suivants font entrer le lecteur au cœur des histoires de vie. Il s'agit d'abord de décrire les alternances emploi/chômage et les mouvements autour du seuil de pauvreté. Une fraction des jeunes étudiés reste bloquée durablement dans des emplois culs-de-sac, souvent de courte durée et peu qualifiés : 
c'est le cycle bas salaire/absence de salaire. En ajoutant à leur échantillon des personnes de plus de 40 ans, le même phénomène est observé. Pour autant, et c'est un enseignement majeur de l'ouvrage, appuyé sur de nombreux exemples et citations, aucun signe de découragement/désengagement n'apparaît : la valeur travail reste forte. En raison de ce résultat, en conclusion de l'ouvrage, les auteurs reviennent, d'un point de vue critique, sur ce qu'ils appellent le "grand mythe » du chômage volontaire et de l'abus des aides sociales. Particulièrement présent dans l'histoire des politiques de workfare au Royaume-Uni, ce grand mythe résonne aussi dans les débats français.

La plupart des interviewés sont au départ sans formation ni diplôme. Beaucoup passent par des stages qu'ils apprécient, notamment pour acquérir des National Vocational Qualifications ${ }^{3}$ de base qui sont néanmoins insuffisantes et inadaptées à la nature des emplois occupés. L'étude des pratiques des agences de l'emploi, agences d'intérim et autres, montre qu'elles sont souvent démunies devant la pénurie d'emplois de qualité et/ou qu'elles contribuent à l'enfermement des demandeurs d'emploi sur le segment des emplois de mauvaise qualité. Cet enfermement s'explique aussi par le fait que les enquêtés s'appuient souvent sur des réseaux relationnels forts (parents, proches) qui appartiennent au même segment.

Lorsque l'on élargit le regard au-delà de l'emploi, ce que permet l'analyse biographique approfondie, il est possible de répertorier plusieurs autres facteurs, classiques mais non toujours pris en charge de façon cohérente dans les politiques publiques. Les problèmes de santé (parfois directement liés à la mauvaise qualité des conditions de travail), les contraintes familiales telles que la garde des enfants ou les soins à des proches, la consommation de drogue, ou la difficulté de l'accès au lieu de travail faute de moyen de transport sont ainsi mis en lumière. Par là, ce sont les relations complexes entre offre et demande de travail qui sont révélées par ce qu'en disent les personnes enquêtées.

11 On peut alors mieux comprendre pourquoi ces travailleurs n'échappent pas à la pauvreté, comment les mailles du filet des aides sociales, rompues par les discontinuités de trajectoire, s'avèrent insuffisantes.

12 En conclusion, outre leur insistance sur le "grand mythe ", les auteurs reviennent sur d'autres travaux, y compris plus quantitatifs, qui permettent de conforter leur approche monographique. Ils avancent l'hypothèse forte du caractère "fonctionnel » de ce nouveau précariat dans une économie ouverte et censée être basée sur l'économie de la connaissance. Dans cette économie, les emplois peu qualifiés, les mauvais emplois demeurent. Ceux qui sont pris dans le cycle emploi de faible qualité/absence de salaire, en passant parfois par la case d'une aide sociale insuffisante pour les sortir de la pauvreté, constituent une forme de nouvelle «armée de réserve». Tout en ne partageant pas totalement les thèses de Guy Standing ${ }^{4}$ qui voit dans la croissance du précariat l'émergence d'une nouvelle classe sociale, les auteurs le rejoignent sur ses principaux attributs, détaillés tout au long de l'ouvrage; envisagés sous l'angle du travail, de l'emploi, du revenu, des qualifications, etc., ils sont aussi liés au recul des filets de sécurité existants. Un tel constat conduit naturellement les auteurs à formuler un ensemble de critiques et de recommandations pour les politiques publiques.

13 On pourra évidemment discuter de la pertinence des choix méthodologiques: se focaliser, zoomer exclusivement sur ceux qui sont le plus dans la difficulté, le faire dans une région particulière et sur un petit échantillon. Deux arguments forts sont opposables à ces critiques : d'une part, le caractère « invisible » de ce segment qui ne se limite ni aux jeunes primo-entrants ni aux chômeurs de longue durée, plus facilement 
identifiables dans les statistiques et pris en compte dans les politiques; d'autre part, la nécessité d'une approche englobant l'ensemble de l'histoire de vie pour analyser les interactions entre travail, emploi et pauvreté.

Pour le lecteur français (qui regrettera de ne pas bénéficier d'une traduction... mais l'inverse est vrai, la littérature française sur cette question est inconnue des auteurs), l'un des intérêts de cet ouvrage réside d'abord dans le recul qu'il permet de prendre sur le succès du Royaume-Uni en termes d'emploi, souvent attribué à la flexibilité de son marché du travail et à l'efficacité de ses politiques de workfare. Le faible taux de chômage et sa décrue rapide dans la période récente masquent l'importance des emplois de mauvaise qualité, de la pauvreté dans l'emploi.

Il incite, par ses entrées pluridimensionnelles, à dépasser l'approche, souvent dominante en France, par les seuls statuts d'emploi (contrat à durée déterminée versus contrat à durée indéterminée) pour prendre la question des emplois à bas niveaux de salaire de manière plus globale. C'était la ligne suivie par Ève Caroli et Jérôme Gautié en 2008 dans le cadre de la recherche internationale sur le travail à bas niveau de salaire menée pour la fondation Russell Sage ${ }^{5}$. Le salaire minimum peut aussi masquer en France une réalité multiforme d'emplois de mauvaise qualité.

Il fait en outre écho à des travaux plus récents, comme ceux de Claude Picart ${ }^{6}$, sur la dualisation croissante du marché du travail en France et sur la frange du salariat enfermée dans l'alternance mauvais emplois, chômage ou inactivité.

Et, ainsi que le concluent les auteurs, il incite à penser non seulement une politique de l'emploi, mais aussi une politique de la qualité du travail, indissociables de celles de la protection sociale.

\section{NOTES}

1. Frazer H., Marlier É. (2010), In-work poverty and labour market segmentation in the EU, Ceps/ Instead, European network of independent experts on social inclusion, $\mathrm{n}^{\circ} 2010-02$; disponible en ligne à l'adresse : www.ceps.lu/publi_viewer.cfm ?tmp =2709; consultée le 23 juin 2015.

2. Le welfare to work (également appelé workfare) désigne les politiques actives de l'emploi conditionnant diverses aides sociales à la recherche active d'un emploi, voire à l'obligation de prendre « n'importe quel » emploi.

3. Les National Vocational Qualifications sont l'une des composantes du système des diplômes professionnels anglais, dont la valeur sur le marché du travail est souvent analysée comme très faible.

4. Standing G. (2011), The precariat : the new dangerous class, London, Bloomsbury Academic.

5. Caroli È., Gautié J. (2008), Low-wage work in France, New York, Russell Sage Foundation.

6. Picart C. (2014), «Une rotation de la main-d'œuvre presque quintuplée en 30 ans : plus qu'un essor des formes particulières d'emploi, un profond changement de leur usage », Emploi et salaires. Insee références, Paris, Insee.

Travail et Emploi, 142 | avril-juin 2015 


\section{AUTEURS}

\section{PHILIPPE MÉHAUT}

Laboratoire d'économie et de sociologie du travail (Lest) 